\title{
Things We Do For No Reason: HIT Testing in Low Probability Patients
}

\author{
Amanda Li, MD'; Lisa K Hicks, MD, MSc²; Eddy Fan, MD, PhD³*
}

'Department of Medicine, University of Toronto, Toronto, Ontario, Canada; ${ }^{2}$ Division of Hematology, St. Michael's Hospital, Toronto, Ontario, Canada; Interdepartmental Division of Critical Care Medicine, University of Toronto, Toronto, Ontario, Canada.

Inspired by the ABIM Foundation's Choosing Wisely ${ }^{\circledR}$ campaign, the "Things We Do for No Reason" series reviews practices which have become common parts of hospital care but which may provide little value to our patients. Practices reviewed in the TWDFNR series do not represent "black and white" conclusions or clinical practice standards, but are meant as a starting place for research and active discussions among hospitalists and patients. We invite you to be part of that discussion.

\section{CLINICAL SCENARIO}

A 59-year-old man with cirrhosis secondary to nonalcoholic steatohepatitis was admitted to the intensive care unit (ICU) for management of hepatorenal syndrome and work-up for liver transplantation. On admission, his platelet count was $90 \times$ 109/L (normal 150-400 × 109/L), and he was started on thromboprophylaxis with unfractionated heparin (UFH) 5,000 units subcutaneously twice daily. His platelet count began to fall two days after admission. He did have a history of prior heparin exposure associated with his hemodialysis sessions in the past 30 days. During this period, he also had an episode of fever, and antibiotics were initiated for a presumed line infection. He also required periodic vasopressor support for hypotension. His platelet count reached $14 \times 109 / \mathrm{L}$ by the end of two weeks. He did not have any symptoms of thrombosis, skin necrosis, or reaction to heparin exposure.

\section{BACKGROUND}

Thrombocytopenia is common, especially during critical illness, occurring in up to $50 \%$ of patients. ${ }^{1}$ In this population, thrombocytopenia is often due to sepsis, hemorrhage, liver dysfunction, and drug reactions. ${ }^{1,2}$ Heparin-induced thrombocytopenia (HIT) is an acquired thrombotic drug reaction resulting from platelet activation secondary to antibodies formed against the heparin-modified platelet factor 4 (PF4) complexes. ${ }^{3}$ This leads to platelet aggregation and dysregulation of the coagulation cascade, which can result in arterial or venous thromboembolic events in up to $50 \%$ of patients. ${ }^{3}$ Mortality associated with HIT can be as high as $30 \%$ in this critically ill population. ${ }^{3}$ Diagnosis of HIT can be made initially through the

*Corresponding Author: Eddy Fan, MD, PhD; E-mail: eddy.fan@uhn.ca; Telephone: 416-340-5483, Twitter: @efan75

Published online first April 8, 2019.

Received: August 24, 2018; Revised: December 27, 2018;

Accepted: January 7, 2019

() 2019 Society of Hospital Medicine DOI 10.12788/jhm.3165 enzyme-linked immunosorbent assay (ELISA). Management of HIT involves immediate cessation of heparin and initiation of therapeutic anticoagulation with nonheparin agents in order to prevent or treat the thrombotic events. ${ }^{4,5}$

The true incidence of HIT remains low, occurring in $0.2 \%$ to $5 \%$ of patients exposed to heparin and less than $1 \%$ in the ICU population. $2,3,6,7$ However, given the high incidence of thrombocytopenia in the ICU, the diagnosis of HIT is often considered, resulting in over-testing in this population. Studies suggest that more than 200 ELISAs are requested per year at many hospitals. ${ }^{8,9}$ This can lead to significant clinical and economic consequences.

\section{WHY YOU MIGHT THINK HIT TESTING WITH ELISA IS HELPFUL}

Thrombocytopenia is common in hospitalized patients while heparin is frequently used for thromboprophylaxis or therapeutic anticoagulation. As a result, a diagnosis of HIT is often considered.' The high stakes of the inpatient environment, coupled with the increased frequency of thrombocytopenia and heparin exposure, has led to increased use of HIT testing in this population. ${ }^{10}$

The most widely available diagnostic test for HIT is the ELISA which detects anti-PF4-heparin antibodies but also nonpathogenic antibodies. ${ }^{11}$ As a result, the ELISA has a sensitivity close to $100 \%$, allowing physicians to rule out HIT if the test is negative, as indicated by an optical density (OD) of less than $0.4{ }^{7}$ Confirmatory testing with the functional serotonin release assay (SRA) is the reference standard as it confers both a high sensitivity and specificity for HIT. ${ }^{11}$ Due to technical aspects, SRA, unlike the ELISA, is not available in every center and is often outsourced to external labs. Turn-around time for external SRA testing can vary from days to weeks versus hours for the ELISA. The cost for SRA is approximately $\$ 120$ (USD) per test compared to $\$ 30$ (USD) per ELISA. Therefore, the ELISA is the recommended initial test due to its quick turn-around time and lower costs. ${ }^{12,13}$ For these reasons, the SRA test should not be used initially, but rather to confirm the diagnosis of HIT in patients with a positive ELISA.

\section{WHY YOU SHOULD NOT TEST LOW PROBABILITY PATIENTS FOR HIT}

The " $4 T$ 's" scoring system is a clinical scoring system that estimates the pretest probability of HIT using clinical and basic laboratory parameters (Table). ${ }^{14}$ The $4 \mathrm{~T}^{\prime}$ s score provides a pretest probability for HIT using four parameters: platelet count, timing of platelet fall, presence of thrombotic events, and the 
TABLE. 4T's Score (Adapted from Cuker et al. Blood 2012;120(20):4160-4167. doi: 10.1182/ blood-2012-07-443051.14)

\begin{tabular}{llll}
\hline 4Ts & 2 Points & 1 Point & 0 Points \\
\hline Thrombocytopenia & Platelet fall $>50 \%$ and platelet nadir $\geq 20 \times 10^{9} / \mathrm{L}$ & Platelet fall $30 \%-50 \%$ or platelet nadir 10-19 $\times 10 \% / \mathrm{L}$ & Platelet fall $<30 \%$ or platelet nadir $<10 \times 109 / \mathrm{L}$ \\
\hline Timing of platelet count fall & $\begin{array}{l}\text { Onset between } 5-10 \text { days or platelet fall } \leq 1 \text { day } \\
\text { (prior heparin exposure within } 30 \text { days) }\end{array}$ & $\begin{array}{l}\text { Consistent with days } 5-10 \text { fall, but not clear } \\
\text { (eg, missing platelet counts) or onset after day 10 or } \\
\text { fall } \leq 1 \text { day (prior heparin exposure 30-100 days ago) }\end{array}$ \\
\hline Thrombosis or other sequalae & $\begin{array}{l}\text { New thrombosis (confirmed); skin necrosis; acute } \\
\text { systemic reaction after intravenous unfractionated } \\
\text { heparin bolus }\end{array}$ & $\begin{array}{l}\text { Progressive or recurrent thrombosis; non-necrotizing } \\
\text { (erythematous) skin lesions; suspected thrombosis } \\
\text { (not proven) }\end{array}$ & None \\
\hline Other causes of thrombocytopenia & None apparent & Possible & Definite \\
\hline
\end{tabular}

likelihood of another cause of thrombocytopenia. Based on these parameters, the pretest probability for HIT can be divided into three categories: low (4T's score of $\leq 3$ ), intermediate (score 4-5), or high (score 6-8). ${ }^{14-16}$

Validation of the $4 T^{\prime}$ 's score has shown that a low probability score carries a negative predictive value of $99 \%$ in a patient population with varying HIT prevalence rates. ${ }^{14}$ Therefore, having a low score is sufficient to rule out HIT without the need for further laboratory testing. ${ }^{14-16}$ Although the HIT ELISA confers high sensitivity, due to its detection of nonpathogenic antibodies, its specificity can range from $74 \%$ to $84 \% .{ }^{15}$ Therefore, in the setting of a low 4T's score, HIT testing is not only unnecessary, it can be harmful due to the risk of treating a false positive result. For instance, assuming an average HIT prevalence of $1 \%$ and a false positive rate of $16 \%$ (specificity $84 \%), 1 / 17$ (5.6\%) patients with a positive ELISA will have HIT if testing is pursued in an indiscriminate manner. The American Society of Hematology Choosing Wisely ${ }^{\circledast}$ Campaign has highlighted this concern by advising physicians that they should "not test or treat for suspected HIT in patients with a low pretest probability of HIT." ${ }^{\text {17 }}$

False positive results on HIT tests are not a trivial concern. The most recognizable adverse event associated with HIT treatment is an elevated risk of bleeding while receiving nonheparin agents. Availability of nonheparin anticoagulants vary by center; however, the most commonly used agents include argatroban, danaparoid, bivalirudin, and off-label fondaparinux. ${ }^{4}$ Due to its short half-life and hepatic clearance, argatroban is commonly used for cases of confirmed or suspected HIT. A retrospective study assessing the bleeding risk of critically ill patients on argatroban therapy suggests a major bleeding risk of $10 \%$ within two days of argatroban initiation. ${ }^{18}$ In addition, factors such as the presence of elevated bilirubin, major surgery, weight $>90 \mathrm{~kg}$, and platelet count $<70 \times 109 / \mathrm{L}$ were found to be associated with increased risk for major bleeding..$^{18}$ These identified risk factors are very common in the inpatient setting. As a result, monitoring and titration of argatroban can be challenging.

Over-diagnosis and over-treatment can also lead to significant costs to the healthcare system. A retrospective study assessing the use of HIT testing found that out of 218 HIT ELISA's sent over a one-year period at a single institution, 161 (74\%) were sent inappropriately (ie, in patients with a low pretest probability), with only one resulting in confirmed HIT by SRA. This incurred an additional cost of $\$ 33,000$ (USD) for testing alone. ${ }^{8} \mathrm{~A}$ retrospective study of 85 patients assessed the costs of treating patients with a false positive HIT assay. They found that the average duration of treatment with a nonheparin agent was three days and the total cost per patient was $\$ 982$ (USD). ${ }^{19}$ Treatment with a nonheparin agent such as argatroban costs more than $\$ 700$ (USD) per day while the continuation of unfractionated heparin for prophylaxis costs less than $\$ 10$ (USD) per day. ${ }^{20}$

Lastly, a diagnosis of HIT can also result in late consequences due to heparin re-exposure. Clinicians may be wary of exposing patients to heparin in situations where heparin may be the most appropriate agent such as cardiovascular surgery, percutaneous interventions, routine thromboprophylaxis, or therapeutic anticoagulation. In these situations when heparin is the agent of choice, determining safety for re-exposure requires further antibody testing which may delay procedures or result in the use of alternative agents with their associated risks and cost implications. ${ }^{4}$

\section{WHEN HIT TESTING WITH ELISA MAY BE HELPFUL}

Laboratory testing for HIT is appropriate when the pretest probability for HIT is intermediate or high based on the 4T's score. ${ }^{14-16}$ Studies assessing the application of the 4T's score have shown that a moderate or high pretest probability carries a probability of having true HIT in $14 \%$ and $64 \%$ of the cases respectively. ${ }^{14}$ However, due to the subjective nature of the 4T's score components, it is important to recognize that in nonexpert hands, the $4 T^{\prime}$ 's scoring system can suffer from a lack of interrater reliability. ${ }^{16}$

As discussed above, a negative ELISA $(O D<0.4)$ helps to rule out HIT and allow heparin to be safely reintroduced without any further testing. If ELISA is positive $(O D \geq 0.4)$ confirmation testing with SRA should be performed. ${ }^{5}$ However, studies suggest that the magnitude of the OD is associated with increased likelihood for true HIT, with an OD of greater than 2.00 associated with a positive SRA approximately $90 \%$ of the time. ${ }^{21}$ This suggests that if OD values are strongly positive ( $\geq 2.00)$, SRA can be deferred. ${ }^{5}$

Due to the SRA limited availability, confirmatory testing is not always possible or in some situations, SRA results may be negative despite a positive OD. In both these cases, discussion with the Hematology service is recommended. 


\section{WHAT WE SHOULD DO INSTEAD OF SENDING ELISA}

When presented with a case of thrombocytopenia, it is important for clinicians to consider a broad approach in their differential diagnosis. Hospitalists should investigate common etiologies, consider the coagulation parameters, liver enzymes, nutritional status, peripheral blood smear, and a detailed history and physical exam to identify other common potential cause such as sepsis.

The 4T's score should be applied in patients who have had recent heparin exposure. A score of $\leq 3$ indicates a low pretest probability; therefore, HIT is unlikely and further testing is not needed. A score of $\geq 4$ indicates an intermediate or high pretest probability and should prompt clinicians to consider further HIT testing with ELISA. In these situations, heparin should be held, and nonheparin agents should be initiated to prevent thromboembolic complications. In their study of ICU patients, Pierce et al. found that $17 \%$ of patients did not have a concurrent cessation of heparin and initiation of alternative agents despite a high clinical suspicion for HIT. ${ }^{1}$ Lastly, if hospitalists have concerns regarding HIT testing or management, expert consultation with the Hematology service is recommended.

\section{RECOMMENDATIONS}

- Consider a broad differential diagnosis when presented with a hospitalized patient with new thrombocytopenia given the low incidence of HIT ( $<5 \%)$.

- Apply the 4T's score in those who have thrombocytopenia and recent heparin exposure. A low scores 4T's score $(\leq 3)$ predicts a low pretest probability and further testing is not required.

- Patients with moderate or high $4 T^{\prime}$ s score $(\geq 4)$ should have the ELISA test. During this time, heparin should be discontinued and nonheparin agents initiated while waiting for test results.

- Confirmatory testing with SRA should be performed for all positive ELISAs; however, they can be deferred in patients with strongly positive OD $(\geq 2.00)$ on ELISA.

\section{CONCLUSION}

In the opening clinical scenario, the 4T's score would have been 2 ( 1 point for the platelet count, 1 point for the platelet count fall after 10 days, 0 points for thrombosis, and 0 points for an alternative cause of thrombocytopenia), indicating a low pretest probability. Further HIT testing should be deferred as the likelihood for HIT is low. In this case, the more likely etiology for his thrombocytopenia would be sepsis. Therefore, heparin can be safely reinitiated once the platelet count recovers. This case helps to illustrate the importance of keeping a broad differential in cases of thrombocytopenia in the hospitalized patient while concurrently applying the 4T's score to determine appropriateness for further HIT testing. Ultimately by choosing wisely, we can help reduce the cost and safety implications of a falsely positive HIT diagnosis.

What do you do?
Do you think this is a low-value practice? Is this truly a "Thing We Do for No Reason"? Let us know what you do in your practice and propose ideas for other "Things We Do for No Reason" topics. Please join in the conversation online at Twitter (\#TWDFNR)/Facebook and don't forget to "Like It" on Facebook or retweet it on Twitter.

Disclosures: The authors report no conflict of interest.

\section{References}

1. Pierce W, Mazur J, Greenberg C, Mueller J, Foster J, Lazarchick J. Evaluation of heparin-induced thrombocytopenia (HIT) laboratory testing and the 4Ts scoring system in the intensive care unit. Ann Clin Lab Sci. 2013:43(4):429-435.

2. Harada MY, Hoang DM, Zaw AA, et al. Overtreatment of heparin-induced thrombocytopenia in the surgical ICU. Crit Care Med. 2017;45(1):28-34. doi: $10.1097 / \mathrm{ccm} .0000000000002002$.

3. Warkentin TE, Sheppard JAI, Heels-Ansdell D, et al. Heparin-induced thrombocytopenia in medical-surgical critical illness. Chest. 2013;144(3):848-858. doi: 10.1378/chest.13-0057.

4. Linkins LA, Dans AL, Moores LK, et al. Treatment and prevention of heparin-induced thrombocytopenia. Chest. 2012;141(2):e495S-e530S. doi: 10.1378/chest.11-2303.

5. Cuker A, Arepally GM, Chong BH, et al. American Society of Hematology 2018 guidelines for management of venous thromboembolism: heparin-induced thrombocytopenia. Blood Adv. 2018;2(22):3360-3392. doi: 10.1182/ bloodadvances.2018024489.

6. Lo GK, Juhl D, Warkentin TE, Sigouin CS, Eichler P, Greinacher A. Evaluation of pretest clinical score (4 T's) for the diagnosis of heparin-induced thrombocytopenia in two clinical settings. J Thromb Haemost. 2006;4(4):759-765. doi: 10.1111/j.1538-7836.2006.01787.x

7. Cuker A, Cines DB. How I treat heparin-induced thrombocytopenia. Blood. 2012;119(10):2209-2218. doi: 10.1182/blood-2011-11-376293.

8. Elmer P, Passero FC, Xavier M. Retrospective Analysis of Heparin-Induced Thrombocytopenia Management at a Large Tertiary Hospital. J Hematol. 2014;3(2):27-33. doi: http://dx.doi.org/10.14740/jh157w.

9. Goldman R, Ustun B, Levine RL. Retrospective cost analysis of testing for HIT antibodies in a community hospital. Blood. 2008;112(11):4544.

10. Cuker A. Heparin-induced thrombocytopenia (HIT) in 2011: an epidemic of overdiagnosis. Thromb Haemost. 2011;106(6):993-994. doi: 10.1160/TH1109-0677.

11. Warkentin TE. Heparin-induced thrombocytopenia in critically ill patients. Semin Thromb Hemost. 2015;41(5):49-60. doi: 10.1055/s-0034-1398381.

12. Caton S, O'Brien E, Pannelay AJ, Cook RG. Assessing the clinical and cost impact of on-demand immunoassay testing for the diagnosis of heparin-induced thrombocytopenia. Thromb Res. 2016;140:155-162. doi: 10.1016/j. thromres.2016.01.025

13. Nanwa N, Mittmann N, Knowles S, et al. The direct medical costs associated with suspected heparin-induced thrombocytopenia. Pharmacoeconomics. 2011:29(6):511-520. doi: 10.2165/11584330-000000000-00000.

14. Cuker A, Gimotty PA, Crowther MA, Warkentin TE. Predictive value of the 4Ts scoring system for heparin-induced thrombocytopenia: a systematic review and meta-analysis. Blood. 2012;120(20):4160-4167. doi: 10.1182/ blood-2012-07-443051

15. Fiorenza MA, Frazee EN, Personett HA, Dierkhising RA, Schramm GE. Assessment of a modified 4T scoring system for heparin-induced thrombocytopenia in critically ill patients. J Crit Care. 2014;29(3):426-431. doi: 10.1016/j. jcrc.2013.12.010

16. Crowther M, Cook D, Guyatt G, et al. Heparin-induced thrombocytopenia in the critically ill: interpreting the 4Ts test in a randomized trial. J Crit Care. 2014;29(3):470.e7-470.e15 doi: 10.1016/j.jcrc.2014.02.004

17. Hicks LK, Bering H, Carson KR, et al. The ASH Choosing Wisely campaign: five hematologic tests and treatments to question. Blood. 2013:122(24):38793883. doi: 10.1182/blood-2013-07-518423.

18. Doepker B, Mount KL, Ryder LJ, Gerlach AT, Murphy CV, Philips GS. Bleeding risk factors associated with argatroban therapy in the critically ill. J Thromb Thrombolysis. 2012;34(4):491-498. doi: 10.1007/s11239-012-0758-y.

19. Marler J, Unzaga J, Stelts S, Oliphant CS. Consequences of treating false positive heparin-induced thrombocytopenia. J Thromb Thrombolysis. 2015;40(4):512-514. doi: 10.1007/s11239-015-1236-0.

20. Fowler RA, Mittmann N, Geerts W, et al. Cost-effectiveness of dalteparin vs unfractionated heparin for the prevention of venous thromboembolism in critically ill patients. JAMA. 2014;312(20):2135-2145. doi: 10.1001/ jama.2014.15101.

21. Warkentin TE, Sheppard JI, Moore JC, Sigouin CS, Kelton JG. Quantitative interpretation of optical density measurements using PF4-dependent enzyme-immunoassays. J Thromb Haemost. 2008;6(8):1304-1312. doi: 10.1111/j.1538-7836.2008.03025.x 